\title{
Protective effects of notoginsenoside R1 on acute lung injury in rats with sepsis
}

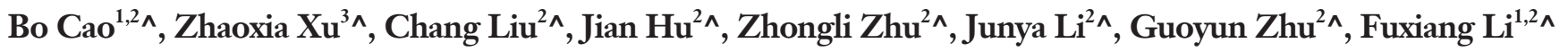 \\ ${ }^{1}$ Southwest Medical University, Luzhou, China; ${ }^{2}$ Department of Intensive Care Medicine, Western Theater General Hospital, Chengdu, China; \\ ${ }^{3}$ Department of Emergency Department, Western Theater General Hospital, Chengdu, China \\ Contributions: (I) Conception and design: B Cao; (II) Administrative support: F Li; (III) Provision of study materials or patients: Z Xu; (IV) Collection \\ and assembly of data: B Cao, C Liu, J Hu, Z Zhu, J Li; (V) Data analysis and interpretation: B Cao, Z Xu, G Zhu; (VI) Manuscript writing: All \\ authors; (VII) Final approval of manuscript: All authors. \\ Correspondence to: Fuxiang Li. Department of Intensive Care Medicine, Western Theater General Hospital, Chengdu, China. Email: lfx98@163.com.
}

Background: To clarify the mechanism of notoginsenoside R1 in the treatment of septic acute lung injury (ALI) based on network pharmacological analysis, and to verify it in the model of septic ALI in rats.

Methods: Based on database searching, the related targets of notoginsenoside R1 and ALI were identified, and the component-disease-target network was constructed. The core targets were screened by proteinprotein interaction (PPI), and the functional enrichment of Gene Ontology (GO) and Kyoto Encyclopedia of Genes and Genomes (KEGG) was analyzed. The rat model of septic ALI was further established to investigate the pharmacological effects of notoginsenoside R1.

Results: Notoginsenoside R1 possibly affected ALI through 150 targets, of which 36 were core targets. GO semantic similarity analysis showed that notoginsenoside R1 might play a role in regulating interleukin 17 (IL-17) signal pathway, tumor necrosis factor (TNF) signal pathway and other key links by regulating MAPK1, MAPK3, IL-1 $\beta$ and other targets. The results of pharmacological experiments showed that notoginsenoside R1 could significantly reduce the wet:dry ratio of the lung in an animal model of ALI, improve the pathological injury of the lung, and reduce the content of IL-1 $\beta$ in serum and in bronchoalveolar lavage fluid (BALF) of experimental animals.

Conclusions: Notoginsenoside R1 can inhibit pulmonary edema, reduce inflammation, and improve lung lesions through multiple targets and pathways to achieve the pharmacological effects in the treatment of septic ALI.

Keywords: Notoginsenoside R1; sepsis; acute lung injury (ALI); network pharmacology; functional enrichment analysis

Submitted Apr 30, 2021. Accepted for publication Jun 11, 2021.

doi: $10.21037 / \mathrm{atm}-21-2496$

View this article at: https://dx.doi.org/10.21037/atm-21-2496

\section{Introduction}

Sepsis is a series of life-threatening dysfunction caused by an uncontrolled response to infection of multiple organs (1). The emergence of acute lung injury (ALI) during sepsis often indicates the systemic dysfunction of multiple organs (2). Owing to the improvement of medical conditions, the ALI mortality rate has been reduced from $60-80 \%$ to $35-40 \%$ in recent years (3). Clinically,

$\wedge$ ORCID: Bo Cao, 0000-0002-3077-1530; Zhaoxia Xu, 0000-0002-9157-9198; Chang Liu, 0000-0001-5310-3845; Jian Hu, 0000-00026670-3089; Zhongli Zhu, 0000-0003-1690-2300; Junya Li, 0000-0003-1647-8968; Guoyun Zhu, 0000-0002-8767-554X; Fuxiang Li, 0000-0003-1434-3568. 
corticosteroids are mainly used to treat ALI, but their use can lead to the risk of infection and other adverse reactions in patients (4). However, there is no recommended ideal solution for ALI.

The heat-clearing and detoxifying effects of traditional Chinese medicine (TCM) are in line with the immune and anti-inflammatory concepts of modern medicine (5). TCM drugs a rich source of drug discovery, most of which do not have serious adverse effects (6). TCM provides theories, resources, and clinical experience for supporting the drug development of ALI (7). Panax notoginseng (Burk) F. H. Chen or notoginseng is a precious medicinal material in China, which is traditionally used in promoting circulation, removing stasis, reducing swelling, and relieving pain. The main active components of notoginseng are total saponins, which have significant anti-inflammatory and immunomodulatory effects (8). Notoginsenoside $\mathrm{R} 1$ is rich in total saponins which may be its main medicinal ingredient. Previous experiments have used lipopolysaccharide (LPS) to induce sepsis in rats or mice (9). Studies have shown that notoginsenoside R1 may protect human lung fibroblast MRC-5 cells from LPS-induced injury (10). This article intends to systematically analyze the mechanism of action of notoginsenoside R1 in the treatment of ALI by means of network pharmacology. The pharmacological effects of notoginsenoside R1 were further verified by replicating the rat model of sepsis-induced ALI. We present the following article in accordance with the ARRIVE reporting checklist (available at https://dx.doi. org/10.21037/atm-21-2496).

\section{Methods}

\section{Experimental materials}

Notoginsenoside R1 reference substance (batch number B2 1099, purity $\geq 98 \%$; Shanghai Yuanye Biotechnology Co., Ltd.); dexamethasone sodium phosphate injection (National Medicines Standard H41020036; Sinopharm Group Sheng Pharmaceutical Co., Ltd.); lipopolysaccharide LPS (batch number S11060; Shanghai Yuanye Biotechnology Co., Ltd.); rat interleukin beta (IL-1 $\beta$ ) enzyme-linked immunosorbent assay kit [ELISA; D731007; Shenggong Bioengineering (Shanghai) Co., Ltd.]; specific-pathogen free (SPF) grade Wistar rats (license number: SCXK (chuan) 2020-030; weight $200 \pm 20 \mathrm{~g}$, half male and half female; Chengdu Dashuo Experimental Animal Co., Ltd.); a fullwavelength microplate reader (Multiskan Sky, Thermo
Fisher Scientific); and a desktop refrigerated centrifuge (Allegra X-15R, purchased from Beckman Coulter, USA) were acquired for this study. Experiments were performed under a project license (No. SXZYYDXDWLL20200233) granted by ethics board of Shaanxi University of Chinese Medicine, in compliance with internationally recognized and institutional guidelines for the care and use of animals.

\section{Screening of targets and construction of relationship networks}

The potential targets of notoginsenoside R1 were screened through Swiss Target Prediction, Pharmapper, BindingDB, and Comparative Toxicogenomics Database (CTD). The molecular targets involved in the pathological progression of lung injury were screened through CTD, GeneCards, and DisGeNet databases using "Lung Injury" and "Acute Lung Injury" as keywords. With reference to literature reports, repeated genes and false-positive genes were discarded. The obtained data were imported into Cytoscape 3.7.2 software to construct a prediction network of a "component-disease-target". A Venn diagram was plotted to confirm the component-disease shared targets.

\section{Protein-protein interaction (PPI) analysis to confirm the core targets}

PPI refers to the process of two or more protein molecules forming a protein complex through noncovalent bonds. The shared targets were analyzed by PPI, and the core targets with a confidence score of more than 0.9 were screened.

\section{Annotation of biological process and function enrichment of core targets}

Further analysis was carried out to show the biological processes and signal pathways that may be involved in the treatment of lung injury by notoginsenoside R1. The Gene Ontology (GO) biological process annotation of the core target (covering biological process, molecular function, and cellular component) and Kyoto Encyclopedia of Genes and Genomes (KEGG) function enrichment were carried out through the "ClusterProfiler" package of R software.

\section{GO semantic similarity analysis}

The similarity of core genes was analyzed through "SemSim" package of R software, and the core functional 
Table 1 Scoring criteria for lung injury

\begin{tabular}{llc}
\hline Lesions & Degree & Score \\
\hline Necrosis of & None & 0 \\
alveolar epithelial & Mild (necrosis of a few cells) & 1 \\
cells & Moderate (small necrosis zone) & 2 \\
& Serve (vast stretches of necrosis) & 3 \\
Inflammatory cell & None & 0 \\
infiltration & Mild (infiltration of a few cells) & 1 \\
& Moderate(infiltration of many cells) & 2 \\
& Serve (massive cell infiltration) & 3 \\
& None & 0 \\
& Mild & 1 \\
& Moderate & 2 \\
& Serve & 3 \\
& None & 0 \\
& Mild & 1 \\
& Moderate & 2 \\
& Severe & 3 \\
\hline
\end{tabular}

genes were further screened by analyzing the functional similarity of gene products.

\section{Animal model}

A total of 60 healthy SPF Wistar rats were used in animal experiments. After 1 week of acclimatization, the rats were allocated into six groups by stratified randomization: a control; model; $5 \mathrm{mg} / \mathrm{kg}, 10 \mathrm{mg} / \mathrm{kg}$, and $20 \mathrm{mg} / \mathrm{kg}$ notoginsenoside R1 groups; and a positive control group (dexamethasone, $2 \mathrm{mg} / \mathrm{kg}$, intraperitoneal injection). There were 10 rats in each group. The rats were administered intragastrically once daily for 7 days before modeling. The control group and the model group were intragastrically administered isopyknic normal saline; 30 minutes after the last administration, LPS $(5 \mathrm{mg} / \mathrm{kg})$ was injected intraperitoneally in all groups except the control group to establish a rat model of sepsis-induced ALI.

\section{Sampling and testing}

After 24 hours of LPS injection, the rats were anesthetized with pentobarbital sodium $(30 \mathrm{mg} / \mathrm{kg})$. Blood was collected from venous plexus of the orbit, centrifuged at 3,500 rpm for 15 minutes at $4{ }^{\circ} \mathrm{C}$, and the serum was collected. The rats were then sacrificed, and tracheotomy was immediately performed so that the left bronchi were tied. The right lung of each rat was washed briefly 3 times with $0.5 \mathrm{~mL}$ of $37^{\circ} \mathrm{C}$ phosphate-buffered saline to collect bronchoalveolar lavage fluid (BALF). The free water was absorbed from the right lung with filter paper before the lung wet:dry weight ratio was measured. The right lung tissue was collected for hematoxylin and eosin (HE) staining, and the pathological changes of the lung tissue were evaluated according to the scoring in Table 1; enzyme-linked immunosorbent assay (ELISA) was applied to show the concentration of inflammatory cytokine IL- $1 \beta$ in BALF and in serum, according to the kit instructions.

\section{Statistical analysis}

SPSS 21.0 software (IBM Corp.) was used for statistical analysis of data. Measurement data are shown as mean \pm $\mathrm{SD}$, and difference between groups was tested by one-way analysis of variance (ANOVA). A $\mathrm{P}$ value $<0.05$ was taken as statistically significant.

\section{Results}

\section{Screening of components, disease, and targets for network relationship construction}

Targets for notoginsenoside R1 were searched for in the databases. Swiss Target Prediction database predicted 17 targets, Pharmapper predicted 189, Binding DB predicted 1 , and CTD predicted 85 . Several databases are included in the definition of the pathological progress of lung injury. CTD yielded 3,300 molecular targets, GeneCards 10, and DisGeNet 20. After deduplication, a total of 3,312 disease targets were obtained. Cytoscape 3.7.2 software was used, and a Venn diagram was generated. Notoginsenoside R1 and ALI shared 150 targets, and the component-disease targets network was constructed (Figure 1).

\section{PPI analysis}

PPI was then applied with the 150 shared targets, and 36 core targets with a confidence score of more than 0.9 were screened. The core targets were mainly inflammationrelated proteins, including STAT3, TNF, IL6, MAPK1, MAPK3, RELA, MAPK8, IL-1ß, IL-10, IL-2, etc. (Figure 2). 


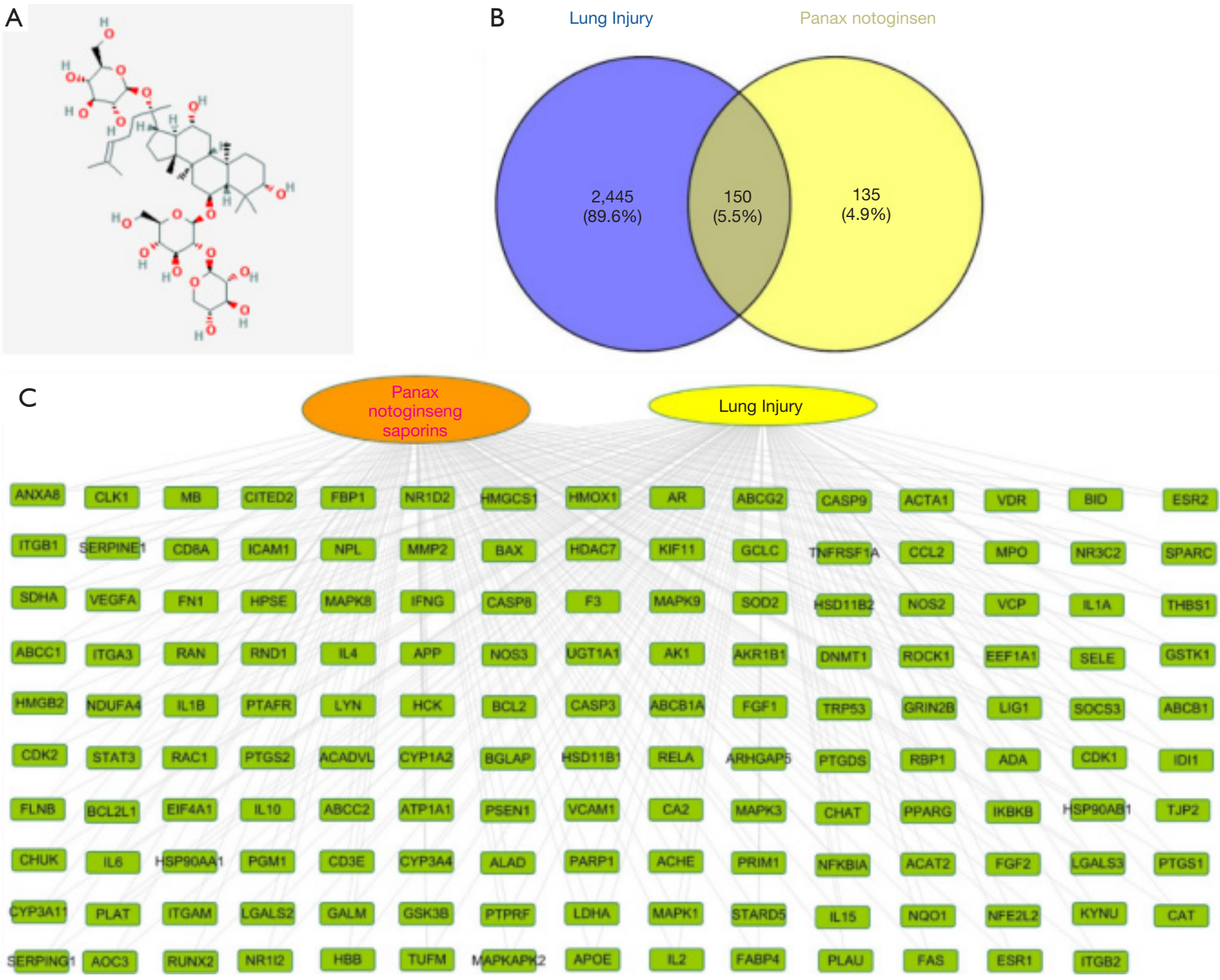

Figure 1 Component-target-disease network of notoginsenoside R1 in ALI. (A) Structure of notoginsenoside R1; (B) Venn diagram of target intersection; (C) component-target-disease network. ALI, acute lung injury.

\section{GO and KEGG functional enrichment of core targets}

GO cell component (CC) enrichment analysis show that the core targets were mainly distributed in lipid rafts, membrane microdomains, membrane region, platelet alpha granule lumen, and platelet alpha granule (Figure 3A). Molecular functions were cytokine receptor binding, cytokine activity, growth factor receptor binding, integrin binding, receptor ligand activity, etc. (Figure 3B). Biological process (BP) mainly involved response to lipopolysaccharide, response to molecule of bacterial origin, positive regulation of establishment of protein localization, regulation of reactive oxygen species metabolic process, and leukocyte migration (Figure 3C). The top 20 related pathways (according to minimum $\mathrm{P}$ value) of KEGG enrichment are shown in Table 2.

The relevant literature indicated that notoginsenoside R1 inhibits sepsis-induced ALI via inactivating inflammation. IL-17, tumor necrosis factor (TNF), human cytomegalovirus infection, Kaposi sarcomaassociated herpesvirus infection, C-type lectin receptor signaling pathway, Th17 cell differentiation, NOD-like receptor signaling pathway, inflammatory bowel disease, necrosis factor $(\mathrm{NF})-\kappa \mathrm{B}$, influenza $\mathrm{A}$, and others play a pharmacological role in the treatment of lung injury with notoginsenoside R1 (Figure 4). 

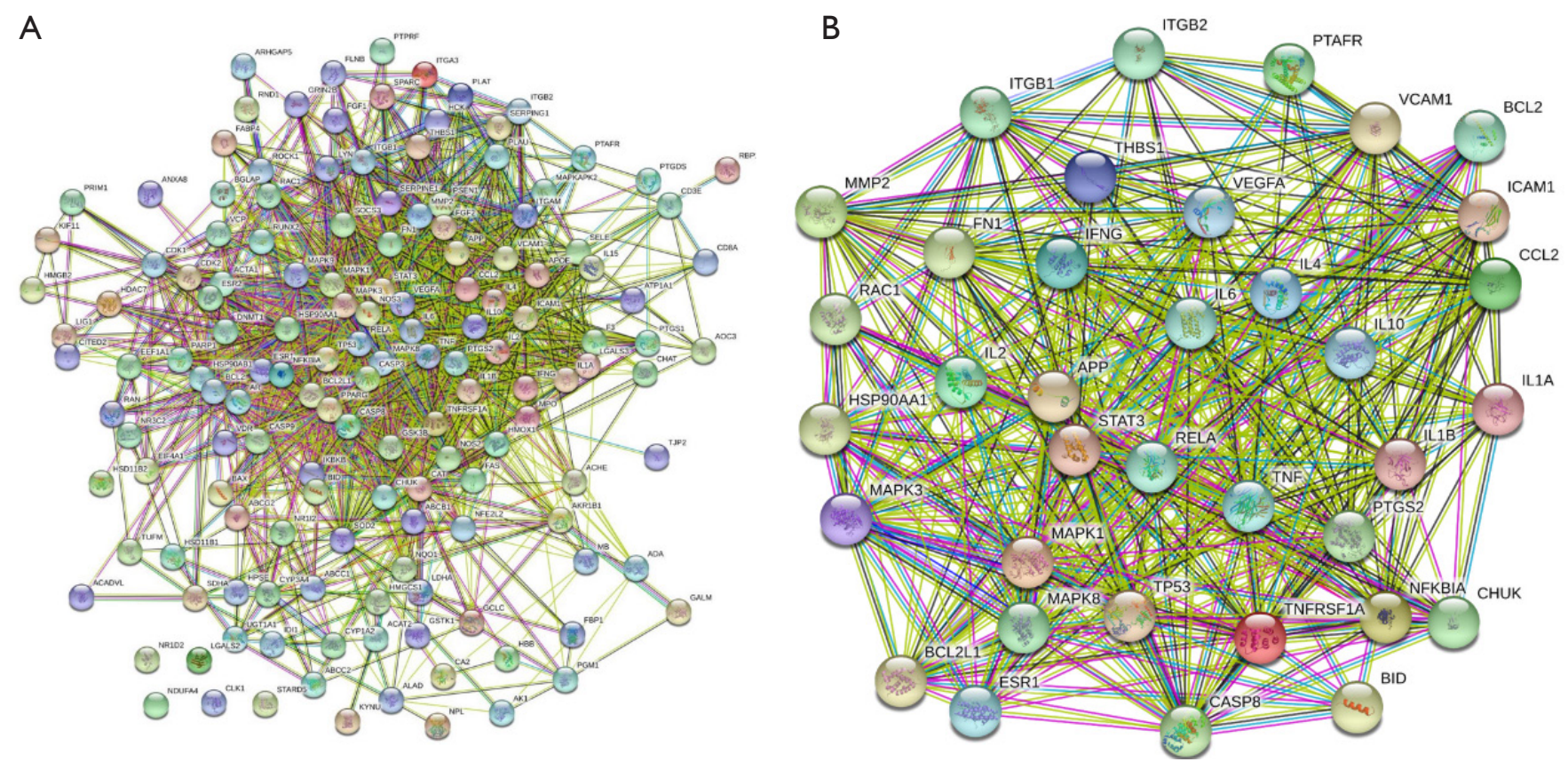

Figure 2 Interactions of the core targets of notoginsenoside R1 in ALI analyzed with PPI. (A) Interactions between the main cotargets; (B) interactions between the core cotargets. ALI, acute lung injury; PPI, protein-protein interaction.

\section{Identifying regulatory genes through gene similarity analysis}

Core genes were further verified by similarity analysis. As shown in Figure 5, MAPK1, MAPK3, IL-1, , CCL2, CHUK, and $B I D$ may be the regulatory genes for notoginsenoside $\mathrm{R} 1$ in treating lung injury.

\section{Notoginsenoside R1 can significantly reduce the wet:dry ratio of lung tissue in LPS-treated rats}

The protective effect of notoginsenoside R1 in sepsisinduced ALI was further validated in LPS-challenged rats. The lung tissue wet:dry ratio indicates the water content of the lungs of experimental animals. It can be seen from Figure 6 that the wet:dry ratio value of the model group was significantly higher than that of the normal control group $(\mathrm{P}<0.05)$; compared with the model group, dexamethasone could significantly reduce the wet:dry ratio value of experimental animals $(\mathrm{P}<0.01)$. Similarly, the wet:dry ratio of notoginsenoside R1 $(5-20 \mathrm{mg} / \mathrm{kg})$ groups decreased significantly compared with the model group $(\mathrm{P}<0.05, \mathrm{P}<0.01)$, indicating that the pulmonary inflammation of each group was weakened with notoginsenoside R1.

\section{Notoginsenoside $R 1$ could significantly improve the pathological conditions of the lungs in LPS-treated rats}

The pathological changes of the lung tissue were observed with HE-stained sections of each rat (Figure 7). The lung tissue of the control group was in good condition, with thin walls, no hyperplasia or thickening of connective tissue, and complete and clear bronchial structures at all levels, with typical alveolar epithelial cell morphology, homogeneous cytoplasmic staining, and clear nuclei (Figure $7 A, B$ ). In the model group (Figure $7 C, D$ ), there was degeneration and necrosis of alveolar epithelial cells with alveolar collapse, narrow alveolar cavity, loss of basic shape of the alveolar epithelial cells, slight edema of the alveolar interstitial, loose connective tissue in the alveolar septum, varying degrees of inflammatory cell infiltration (mainly lymphocytes, neutrophils, or macrophages), and bronchial and perivascular interstitial edema with inflammatory cell infiltration. Unequal amounts of red blood cell infiltration could also be seen in the necrotic area. In the $5 \mathrm{mg} / \mathrm{kg}$ notoginsenoside R1 group (Figure $7 E, F$ ), the lung lesions were observed with relatively thick walls. In 5 of all the 6 rats, alveolar epithelial cell degeneration, necrosis, pyknic or lytic of necrotic cell nucleus, dissolution or disappearance of cytoplasm, pink-stained plasma of the necrotic area, and 
A

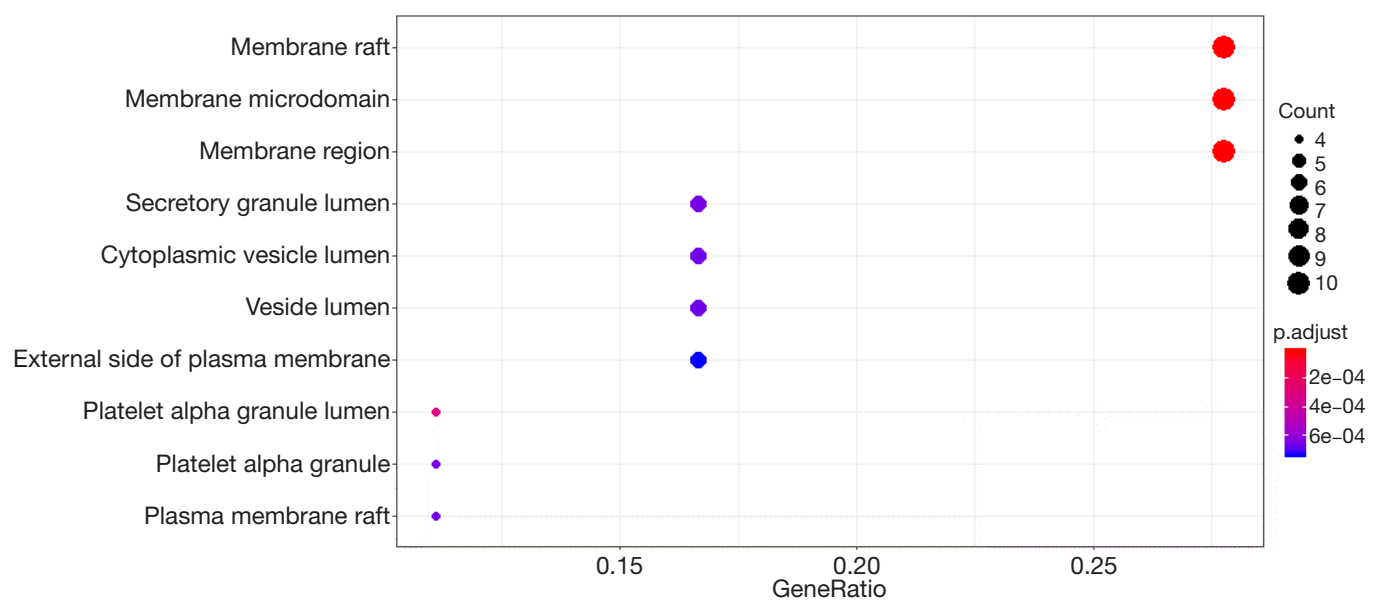

B Positive regulation of establishment of protein localization Response to lipopolysaccharide Response to molecule of bacterial originLeukocyte migration Leukocyte cell-cell adhesion Regulation of reactive oxygen species metabolic process Extrinsic apoptotic signaling pathway

Cellular response to biotic stimulus Cellular response to lipopolysaccharide Regulation of reactive oxygen species biosynthetic process

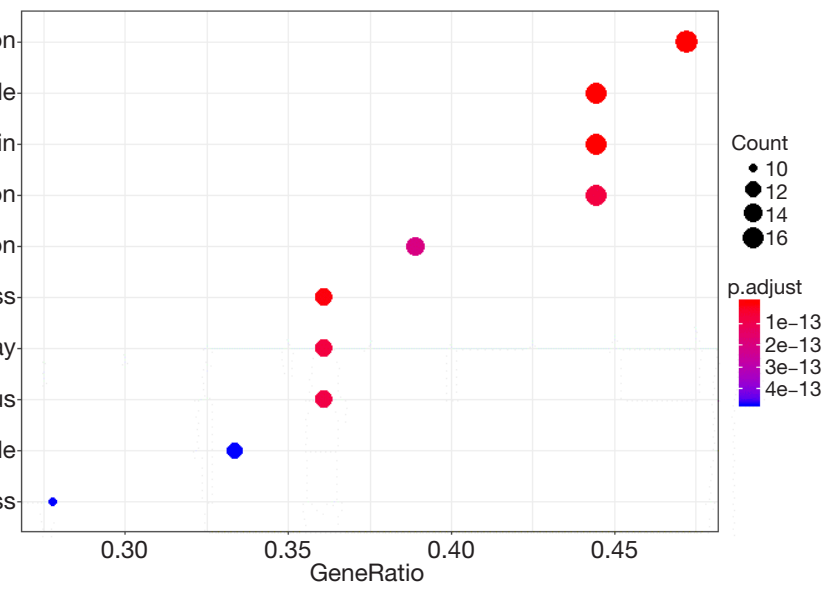

C

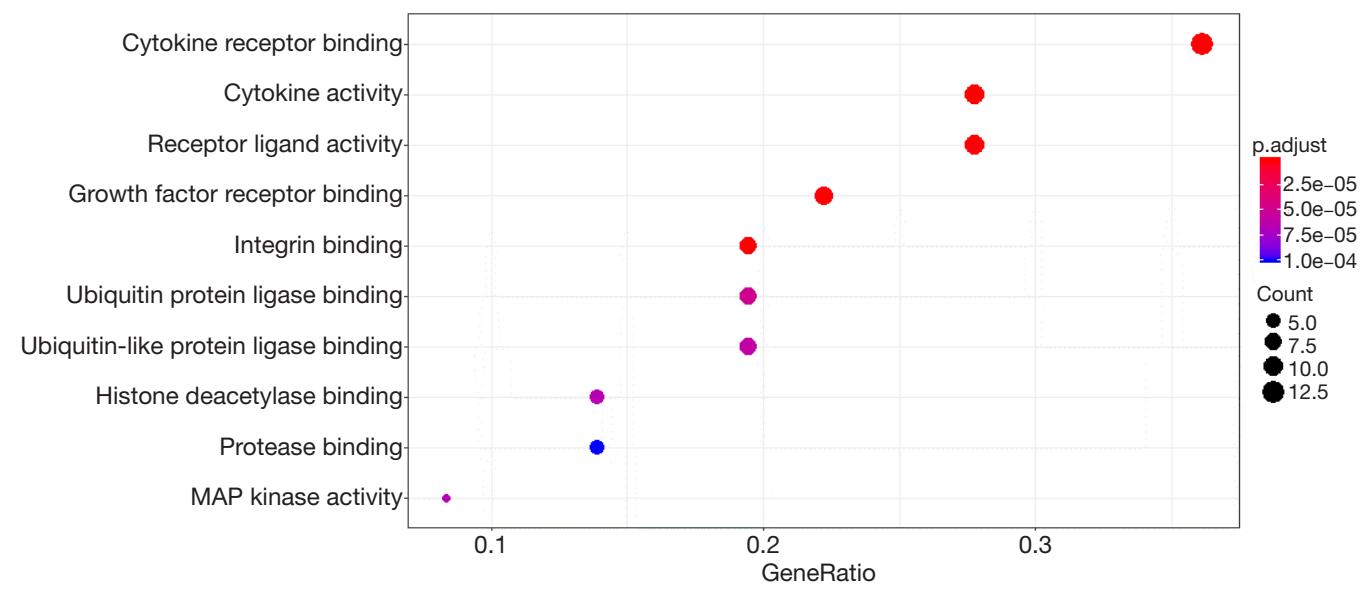

Figure 3 GO enrichment of the core target of notoginsenoside R1 in ALI. (A) Cellular localization, CC enrichment; (B) molecular function, MF enrichment; (C) biological process, BP enrichment. GO, Gene Ontology; ALI, acute lung injury.

cell aggregation (lymphocytes, neutrophils, or macrophages) were seen. In 3 rats, the distribution of red blood cells was seen in the alveoli and alveolar cavity, and edema was seen around the bronchus and vessels, with inflammatory cell infiltration. In the $10 \mathrm{mg} / \mathrm{kg}$ notoginsenoside $\mathrm{R} 1$ group (Figure $7 G, H$ ), the lung tissue structure was relatively normal, 2 rats had alveolar epithelial cell degeneration and necrosis, the morphology and structure of necrotic cells 
Table 2 KEGG related information of core genes involved in regulation

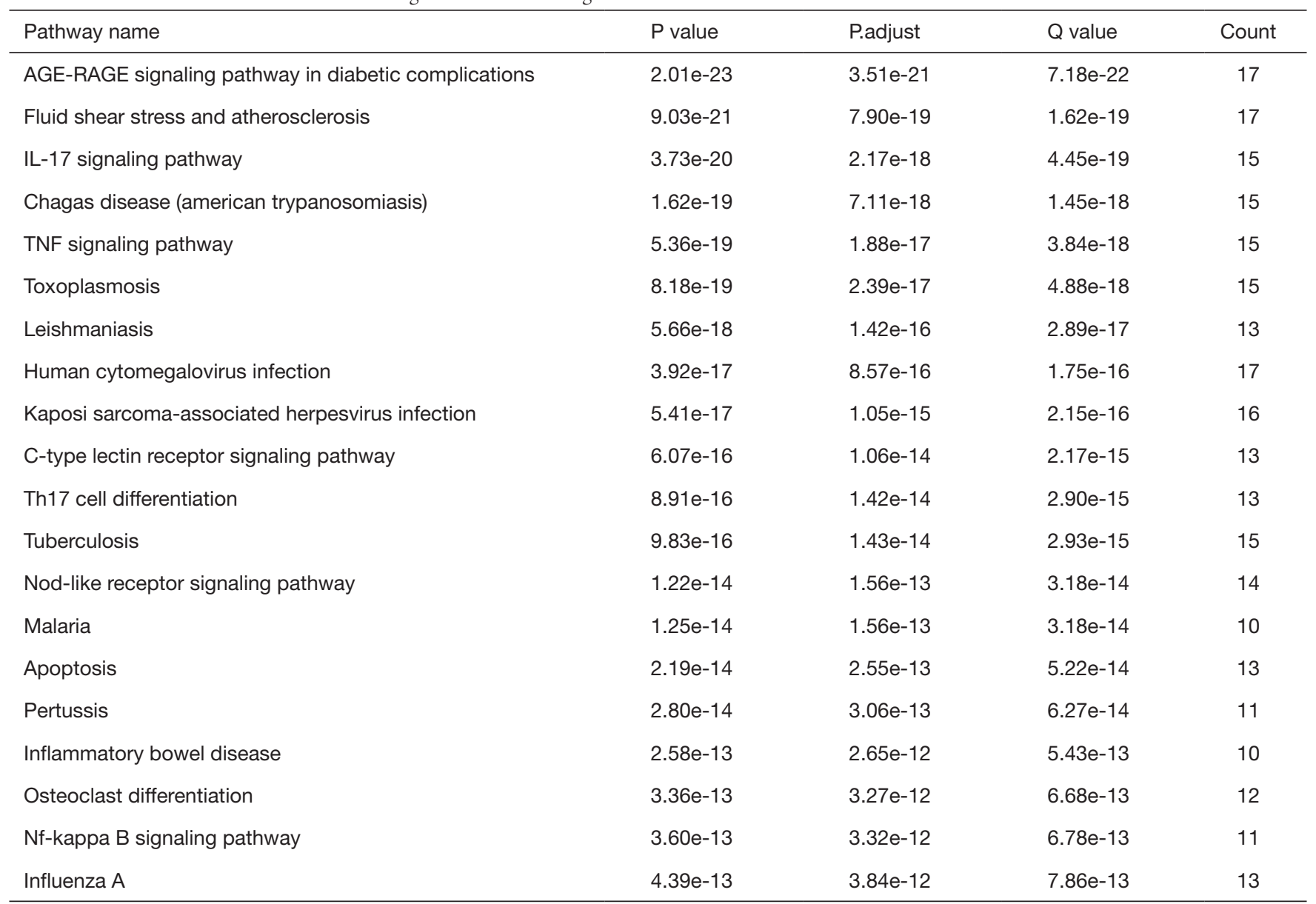

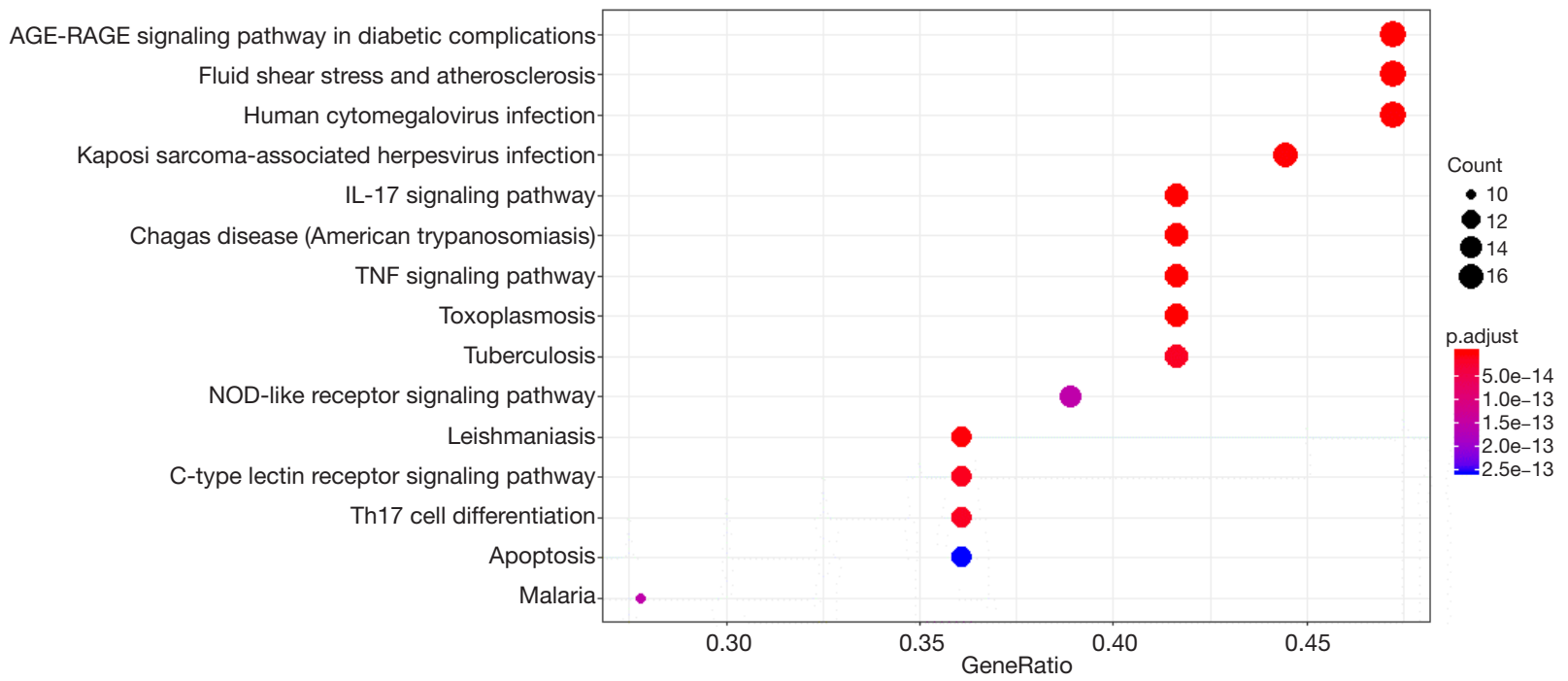

Figure 4 KEGG enrichment of the core target of Notoginsenoside R1 in ALI. KEGG, Kyoto Encyclopedia of Genes and Genomes; ALI, acute lung injury. 


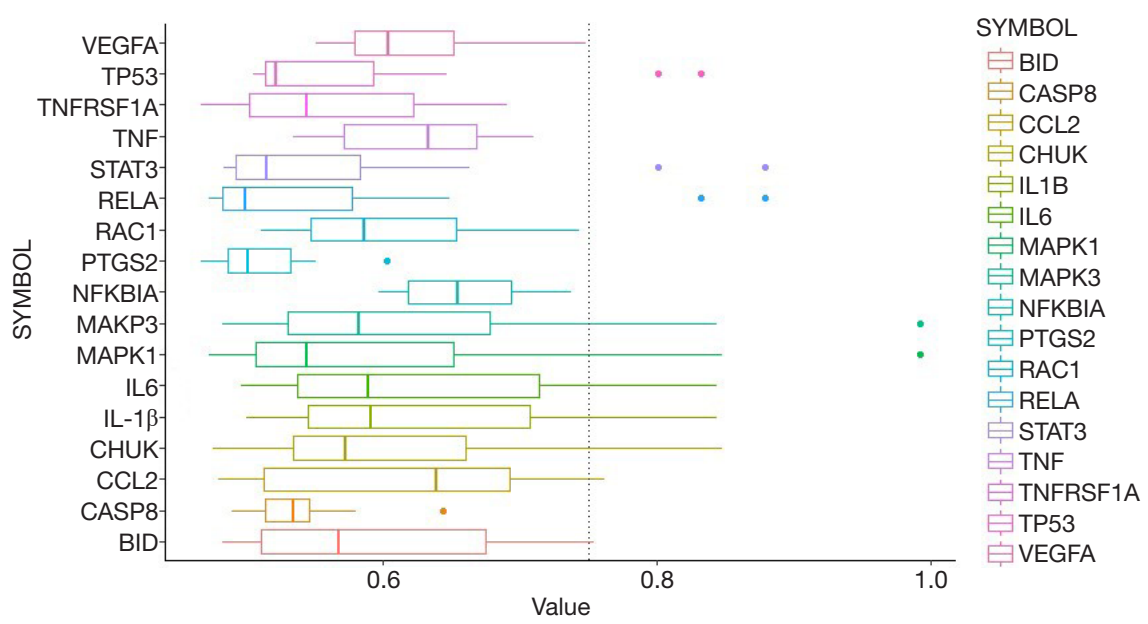

Figure 5 GO semantic similarity analysis of core targets of notoginsenoside R1 in ALI. GO, Gene Ontology; ALI, acute lung injury.

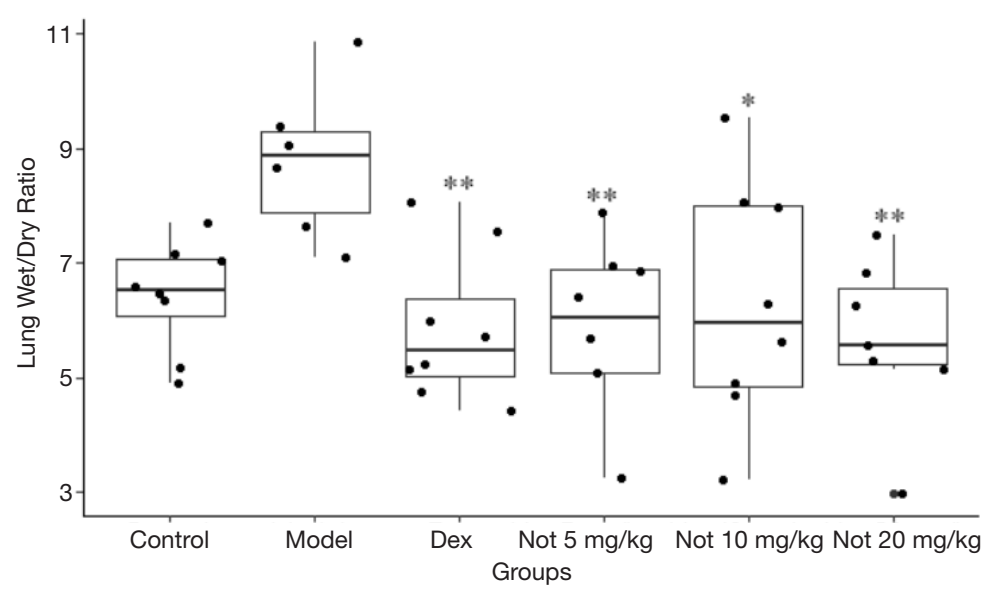

Figure 6 Effects of notoginsenoside R1 on lung edema of LPS-induced ALI rats. The wet:dry ratio was measured. N=6. *, P<0.05; **, $\mathrm{P}<0.01$ vs. model. LPS, lipopolysaccharide; ALI, acute lung injury.

were blurred, and 2 rats had mild alveolar interstitial edema. In all 6 rats, a small amount of lymphocytes, neutrophils or macrophages were seen in the alveolar cavity, and 2 rats had slight bleeding in the necrotic area. The $20 \mathrm{mg} / \mathrm{kg}$ notoginsenoside R1 group was quite normal (Figure 7I,7). Of the 6 rats, 4 showed a small amount of lymphocytes, neutrophils, or macrophages in the alveolar cavity, but no further abnormality was observed. The lung tissue structure of the positive control group was relatively good, with 3 rats having slight edema in the alveolar interstitium, as shown in Figure $7 K, L$.

The HE staining was scored according to the system presented in Table 1. As shown in Table 3, compared with the control group, the lung injury of the model group was significantly decreased $(\mathrm{P}<0.05)$. Compared with the model group, the lung lesion was significantly reduced in the 10 and $20 \mathrm{mg} / \mathrm{kg}$ notoginsenoside R1 groups, as well as the positive control group.

\section{Notoginsenoside R1 reduced IL-1ß levels in LPS-induced ALI rats}

As shown in Figure $8 A$, the serum IL-1 $\beta$ content of the model group after lung injury was significantly higher than that of the normal control group $(\mathrm{P}<0.05)$. Compared with the model group, the positive treatment dexamethasone could significantly reduce the serum IL- $1 \beta$ in rats $(\mathrm{P}<0.05)$, and the serum IL- $1 \beta$ content of rats in the $20 \mathrm{mg} / \mathrm{kg}$ 

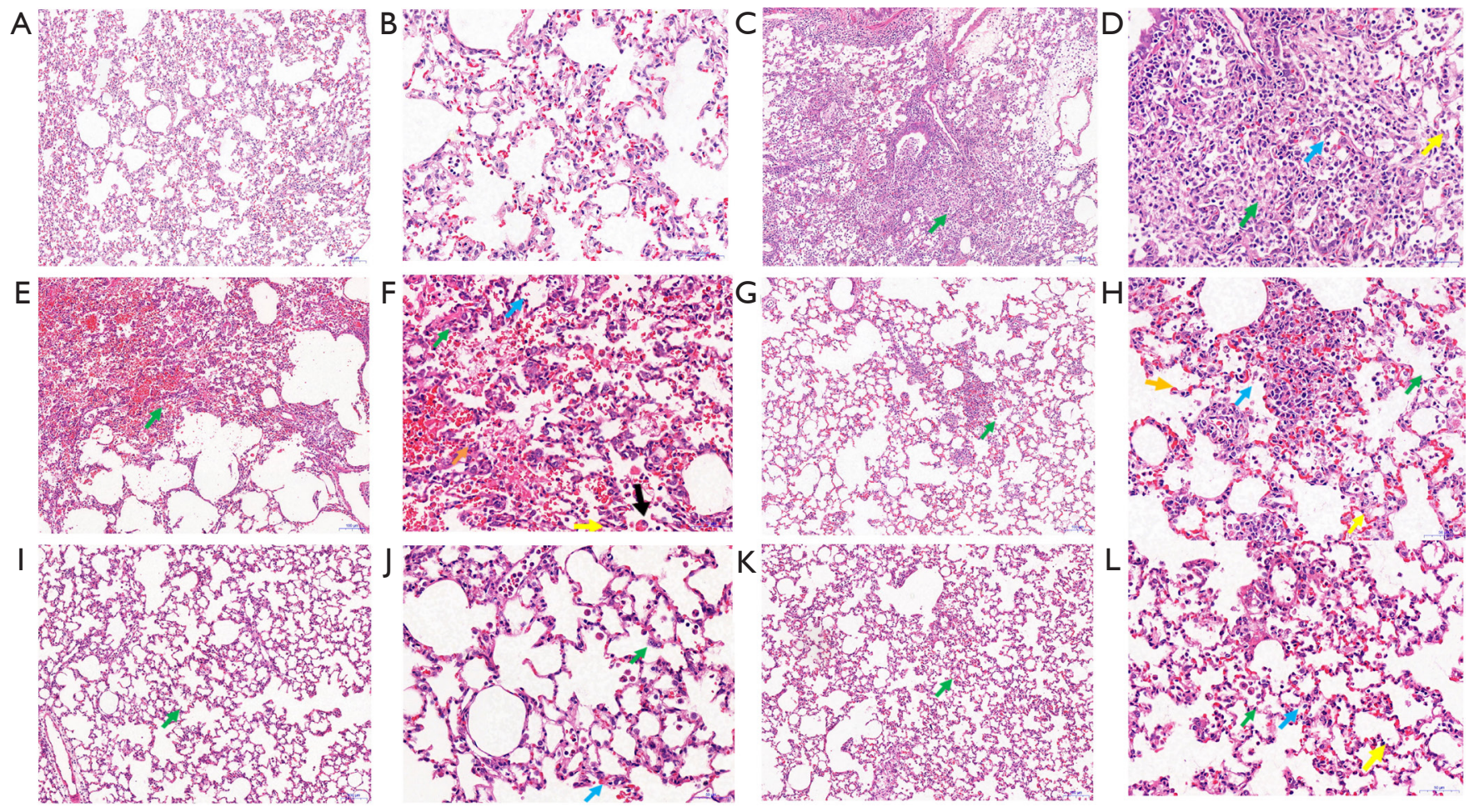

Figure 7 Effects of notoginsenoside R1 on histopathology of LPS-induced ALI rats. HE staining. (A) Control ( $\times 100)$. (B) Control ( $\times 400)$. (C) Model ( $\times 100)$ : alveolar necrosis with inflammatory cell infiltration. (D) Model ( $\times 400)$. (E) Notoginsenoside R1 5 mg/ $\mathrm{kg}$ (×100). (F) Notoginsenoside R1 $5 \mathrm{mg} / \mathrm{kg}$ (×400). (G) Notoginsenoside R1 $10 \mathrm{mg} / \mathrm{kg}(\times 100)$. (H) Notoginsenoside R1 $10 \mathrm{mg} / \mathrm{kg}$ (×400). (I) Notoginsenoside R1 $20 \mathrm{mg} / \mathrm{kg}(\times 100)$. (J) Notoginsenoside R1 $20 \mathrm{mg} / \mathrm{kg}(\times 400)$. (K) Dexamethasone $2 \mathrm{mg} / \mathrm{kg}(\times 100)$. (L) Dexamethasone $2 \mathrm{mg} / \mathrm{kg}(\times 400)$. ( $\uparrow)$ Alveolar necrosis with inflammatory cell infiltration, ( ) neutrophil, ( $\uparrow)$ leukomonocyte, $(\uparrow)$ macrophage, ( $\uparrow$ red blood cells. N=6. LPS, lipopolysaccharide; ALI, acute lung injury; HE, hematoxylin and eosin;

Table 3 Scoring of lung injury

\begin{tabular}{llc}
\hline Group & N & Score \\
\hline Control & 6 & $0.00 \pm 0.00$ \\
Model & 6 & $4.83 \pm 1.72^{\star}$ \\
Notoginsenoside R1 $5 \mathrm{mg} / \mathrm{kg}$ & 6 & $3.67 \pm 1.97$ \\
Notoginsenoside R1 $10 \mathrm{mg} / \mathrm{kg}$ & 6 & $2.33 \pm 0.52^{\triangle}$ \\
Notoginsenoside R1 $20 \mathrm{mg} / \mathrm{kg}$ & 6 & $1.17 \pm 1.47^{\triangle}$ \\
Positive control & 6 & $1.17 \pm 0.75^{\triangle}$ \\
\hline
\end{tabular}

Data are showed as mean $\pm \mathrm{sd} ; \mathrm{n}=6 ;{ }^{*}, \mathrm{P}<0.05$ vs. Control; ${ }^{\Delta}$, $\mathrm{P}<0.05$ vs. Model.

notoginsenoside R1 group was significantly decreased $(\mathrm{P}<0.05)$. As shown in Figure $8 B$, the IL-1 $\beta$ content in BALF of the LPS-induced ALI rats was significantly higher than that in the normal control group $(\mathrm{P}<0.05)$. Compared with model group, dexamethasone activity in the $10 \mathrm{mg} / \mathrm{kg}$ notoginsenoside R1 group significantly reduced the IL-1 $\beta$ content in BALF $(\mathrm{P}<0.05)$.

\section{Discussion}

ALI is the most common in intensive care units. An epidemiological survey study included 2,322 sepsis patients in 44 intensive care units in my country. The results showed that $68.2 \%$ of sepsis patients had ALI, and the 90 -day case fatality rate reached $35.5 \%$ (11).

Even patients who get better and are discharged from the hospital will continue to suffer from the sequelae of critical illness for the next two years or even longer, including exercise restriction, psychological sequelae, reduced quality of life, and financial burden (12). The pathological changes of sepsis ALI mainly include three stages: early inflammatory exudation, subacute tissue hyperplasia and late fibrosis. After effective treatment, most 

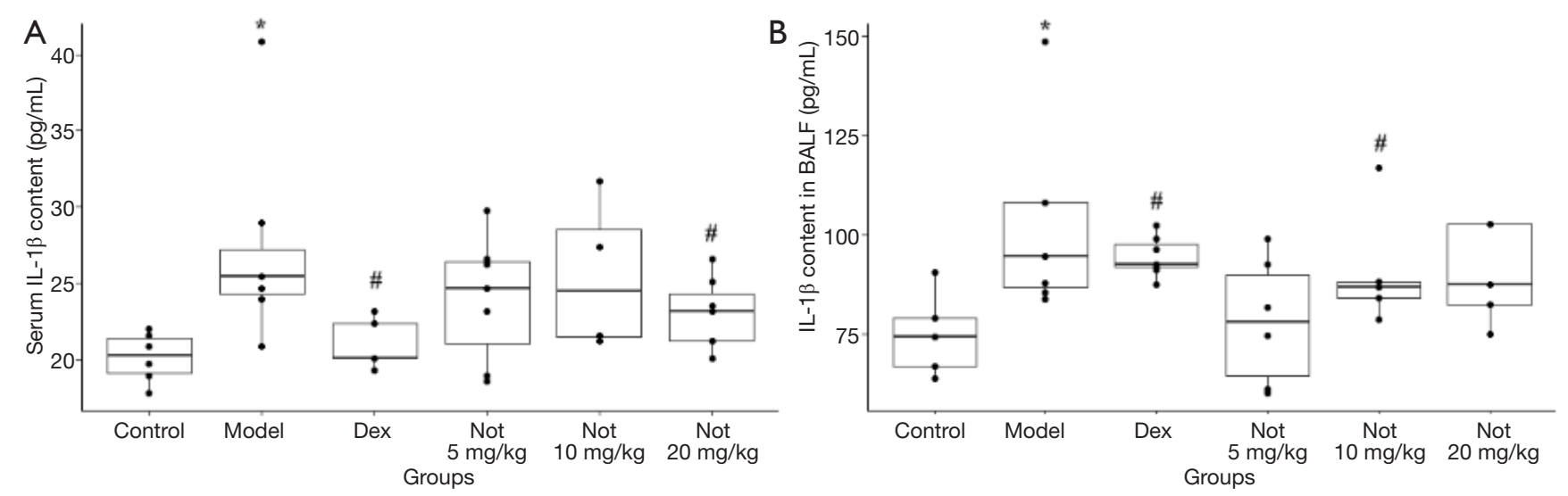

Figure 8 Effects of notoginsenoside R1 on IL-1 $\beta$ level in LPS-induced ALI rats. (A) IL-1 $\beta$ level in serum; (B) IL-1 $\beta$ level in BALF. N=6, *, $\mathrm{P}<0.05$ vs. control; ", $\mathrm{P}<0.05$ vs. model. LPS, lipopolysaccharide; ALI, acute lung injury.

patients will experience the resolution of inflammation and the slow absorption of edema, which is the performance of the first two stages (13). Without early intervention, when the septic shock stage progresses, lung tissue perfusion gradually decreases, pulmonary capillaries contract, and the imbalance of lung ventilation/pulmonary perfusion increases, which often causes irreversible damage to organs and even death. The pathogenesis of sepsis ALI is mainly caused by vascular endothelial injury and alveolar epithelial injury, and its treatment is mainly carried out from two aspects: reducing vascular endothelial injury, protecting and promoting repair of alveolar epithelium.

In the development of sepsis, lung injury often appears first (2). The common features of sepsis-induced ALI are lung epithelial-vascular endothelial cell injury, inflammatory cell infiltration, and pulmonary edema (14). Saponins are the main active ingredients of Panax notoginseng, among which ginsenosides $\mathrm{Rg} 1, \mathrm{Rb} 1$, and notoginsenoside $\mathrm{R} 1$ are the main active monomers (15). These active ingredients have broad bioactivity, including in the cerebral and cardiovascular system (16), liver (17), kidney (18), and bone; and showed significant pharmacological intervention activities in other system diseases (19). In recent years, the academic community has paid increasing attention to its immunomodulatory activity $(8,20)$, and relevant studies have demonstrated that it has a significant therapeutic effect on acute lung injury (21).

Based on the pharmacological activity of notoginsenoside $\mathrm{R} 1$, this paper investigated its mechanism of action on ALI in sepsis by means of network pharmacology. The network pharmacology results showed that notoginsenoside R1 could mainly regulate targets such as MAPK1, MAPK3,
IL-1 $\beta$, CCL2, CHUK, and BID. Notoginsenoside R1 could also regulate IL-17, TNF, human cytomegalovirus infection, Kaposi Sarcoma-associated herpes virus infection, and the C-type lectin receptor signaling pathway to combat sepsis-induced ALI.

The network pharmacology results were confirmed in sepsis-induced ALI rats that were treated with LPS. Notoginsenoside R1 was shown to inhibit pulmonary edema in the process of ALI by reducing the wet:dry ratio of the lungs. By inhibiting the degeneration and necrosis of alveolar epithelial cells, and reducing nuclear shrinkage or lysis, notoginsenoside R1 exerted protective effects on lung epithelial cells and epithelial-vascular cells. The antiinflammatory effects of notoginsenoside R1 were possibly related to the reduced the expression of inflammatory cytokine IL-1 $\beta$ in serum and in BALF, by which ALIrelated inflammation could be inhibited.

The limitation of this study is the lack of studies on the systemic pharmacological mechanism of action of notoginsenoside R1 in the treatment of ALI. The followup research process will need to focus on pivotal signal pathways, and verify the related mechanism of action at the molecular level of genes and proteins.

In conclusion, this study showed that notoginsenoside R1 alleviated sepsis-related ALI by reducing secretion of inflammatory cytokine IL- $1 \beta$ in vivo. The predicted targets of notoginsenoside R1 in inhibiting ALI mainly are inflammation related and included IL-17, TNF, human cytomegalovirus infection, Kaposi sarcoma-associated herpes virus infection, C-type lectin receptor signaling pathway, Th17 cell differentiation, NOD-like receptor signaling pathway, inflammatory bowel disease, NF-кB, 
influenza $\mathrm{A}$, and others.

\section{Acknowledgments}

Funding: None.

\section{Footnote}

Reporting Checklist: The authors have completed the ARRIVE reporting checklist. Available at https://dx.doi. org/10.21037/atm-21-2496

Data Sharing Statement: Available at https://dx.doi. org/10.21037/atm-21-2496

Conflicts of Interest: All authors have completed the ICMJE uniform disclosure form (available at https://dx.doi. org/10.21037/atm-21-2496). The authors have no conflicts of interest to declare.

Ethical Statement: The authors are accountable for all aspects of the work in ensuring that questions related to the accuracy or integrity of any part of the work are appropriately investigated and resolved. Experiments were performed under a project license (No. SXZYYDXDWLL20200233) granted by ethics board of Shaanxi University of Chinese Medicine, in compliance with internationally recognized and institutional guidelines for the care and use of animals.

Open Access Statement: This is an Open Access article distributed in accordance with the Creative Commons Attribution-NonCommercial-NoDerivs 4.0 International License (CC BY-NC-ND 4.0), which permits the noncommercial replication and distribution of the article with the strict proviso that no changes or edits are made and the original work is properly cited (including links to both the formal publication through the relevant DOI and the license). See: https://creativecommons.org/licenses/by-nc-nd/4.0/.

\section{References}

1. Cerceo E, Rachoin JS, Gaughan J, et al. Association of gender, age, and race on renal outcomes and mortality in patients with severe sepsis and septic shock. J Crit Care 2021;61:52-6.

2. Weiss SL, Asaro LA, Flori HR, et al. Multiple Organ Dysfunction in Children Mechanically Ventilated for Acute
Respiratory Failure. Pediatr Crit Care Med 2017;18:319-29.

3. Kost-Alimova M, Sidhom EH, Satyam A, et al. A High-

Content Screen for Mucin-1-Reducing Compounds Identifies Fostamatinib as a Candidate for Rapid Repurposing for Acute Lung Injury. Cell Rep Med 2020;1:100137.

4. Mokra D, Mikolka P, Kosutova P, et al. Corticosteroids in Acute Lung Injury: The Dilemma Continues. Int J Mol Sci 2019;20:4765.

5. Wang Q, Su CP, Zhang HM, et al. Anti-inflammatory mechanism of heat-clearing and detoxifying Chinese herbs. Zhongguo Zhong Yao Za Zhi 2018;43:3787-94.

6. Liu R, Li X, Huang N, et al. Toxicity of traditional Chinese medicine herbal and mineral products. Adv Pharmacol 2020,87:301-46.

7. He YQ, Zhou CC, Yu LY, et al. Natural product derived phytochemicals in managing acute lung injury by multiple mechanisms. Pharmacol Res 2021;163:105224.

8. Zhou Z, He M, Zhao Q, et al. Panax notoginseng saponins attenuate neuroinflammation through TXNIP-mediated NLRP3 inflammasome activation in aging rats. Curr Pharm Biotechnol 2020. [Epub ahead of print]. doi: 10.21 74/1389201021999201110204735.

9. Fisher AB, Dodia C, Chatterjee S, et al. A Peptide Inhibitor of NADPH Oxidase (NOX2) Activation Markedly Decreases Mouse Lung Injury and Mortality Following Administration of Lipopolysaccharide (LPS). Int J Mol Sci 2019;20:2395.

10. Cong S, Xiang L, Yuan X, et al. Notoginsenoside R1 upregulates microRNA-132 to protect human lung fibroblast MRC-5 cells from lipopolysaccharide-caused injury. Int Immunopharmacol 2019;68:137-44.

11. Xie J, Wang H, Kang Y, et al. The Epidemiology of Sepsis in Chinese ICUs: A National Cross-Sectional Survey. Crit Care Med 2020;48:e209-e218.

12. Herridge MS, Tansey CM, Matté A, et al. Functional disability 5 years after acute respiratory distress syndrome. N Engl J Med 2011;364:1293-304.

13. Mowery NT, Terzian WTH, Nelson AC. Acute lung injury. Curr Probl Surg 2020;57:100777.

14. Kumar V. Pulmonary Innate Immune Response Determines the Outcome of Inflammation During Pneumonia and Sepsis-Associated Acute Lung Injury. Front Immunol 2020;11:1722.

15. Wang Q, Mu RF, Liu X, et al. Steaming Changes the Composition of Saponins of Panax notoginseng (Burk.)

F.H. Chen That Function in Treatment of Hyperlipidemia and Obesity. J Agric Food Chem 2020,68:4865-75. 


\section{Page 12 of 12}

16. Liu H, Lu X, Hu Y, et al. Chemical constituents of Panax ginseng and Panax notoginseng explain why they differ in therapeutic efficacy. Pharmacol Res 2020;161:105263.

17. Chen Z, Li C, Yang C, et al. Lipid Regulation Effects of Raw and Processed Notoginseng Radix Et Rhizome on Steatotic Hepatocyte L02 Cell. Biomed Res Int 2016;2016:2919034.

18. Fan C, Chen Q, Ren J, et al. Notoginsenoside R1 Suppresses Inflammatory Signaling and Rescues Renal Ischemia-Reperfusion Injury in Experimental Rats. Med Sci Monit 2020;26:e920442.

19. Liu H, Yang J, Yang W, et al. Focus on Notoginsenoside

Cite this article as: Cao B, Xu Z, Liu C, Hu J, Zhu Z, Li J, Zhu G, Li F. Protective effects of notoginsenoside R1 on acute lung injury in rats with sepsis. Ann Transl Med 2021;9(12):996. doi: 10.21037/atm-21-2496

\section{Cao et al. Protective of notoginsenoside R1 on acute lung injury}

R1 in Metabolism and Prevention Against Human Diseases. Drug Des Devel Ther 2020;14:551-65.

20. Liu S, Yang Y, Qu Y, et al. Structural characterization of a novel polysaccharide from Panax notoginseng residue and its immunomodulatory activity on bone marrow dendritic cells. Int J Biol Macromol 2020;161:797-809.

21. Chen YQ, Rong L, Qiao JO. Anti-inflammatory effects of Panax notoginseng saponins ameliorate acute lung injury induced by oleic acid and lipopolysaccharide in rats. Mol Med Rep 2014;10:1400-8.

(English Language Editor: J. Gray) 